\title{
Anterior cruciate ligament reconstruction with a novel porcine xenograft: the initial Italian experience
}

\author{
STEFANO ZAFFAGNINI, ALBERTO GRASSI, GIULIO MARIA MARCHEGGIANI MUCCIOLI, \\ TOMMASO ROBERTI DI SARSINA, FEDERICO RAGGI, ANDREA BENZI, MAURILIO MARCACCI
}

The II Orthopaedic and Traumatology Clinic, Biomechanics Laboratory and Technological Innovation, Rizzoli Orthopaedic Institute, Bologna, Italy

\begin{abstract}
At the current state of the art in anterior cruciate ligament (ACL) reconstruction, multiple techniques have been presented but none has given clearly defined and improved results.

One of the main issues concerns the choice of graft. The concept of using xenograft tissue, defined as a graft tissue from one species and destined for implantation in an unlike species, was introduced in order to try to overcome the mechanical and biological concerns associated with synthetic materials and the safety and quality concerns and availability problems of allograft tissue. Xenograft tissue carries the risk of producing an immunological reaction. In order to try to overcome or attenuate the immune response against porcine xenograft tissue, the Z-Process ${ }^{\circledR}$ (Aperion Biologics Inc, San Antonio, Texas, USA) has been developed and used to produce the Z-Lig $₫$ family of devices for ACL reconstruction procedures. Z-Lig ${ }^{\circledR}$ is a tendon graft with or without bone blocks, sourced from animal tissue in a manner consistent with what has normally been sourced from human tissue, and processed to overcome anti-Gal-mediated rejection and to attenuate other immunological recognition in humans. All this while ensuring sterility, viral inactivation and preservation of mechanical proprieties appropriate for an ACL reconstruction device. The Z-Lig ${ }^{\circledR}$ device has been tested in skeletally mature monkeys and given interesting and promising
\end{abstract}

\section{Corresponding Author:}

Alberto Grassi, MD

The II Orthopaedic and Traumatology Clinic,

Biomechanics Laboratory and Technological Innovation,

Rizzoli Orthopaedic Institute

Via di Barbiano 1/10, 40136 Bologna, Italy

e-mail: alberto.grassi@ior.it results from the preclinical performance and safety profile point of view. On this basis, it was possible to proceed with the first clinical trial involving humans, which gave similar encouraging results.

The Z-Lig ${ }^{\circledR}$ device has also been implanted in Italy at the Rizzoli Orthopaedic Institute in Bologna, as a part of international multicenter prospective randomized blinded controlled study aimed at comparing xenograft with allograft tissue.

Key Words: anterior cruciate ligament, reconstruction, knee, xenograft, Z-Lig.

\section{Background}

The anterior cruciate ligament (ACL) is the main stabilizer of the knee joint, as it controls anteroposterior and rotatory knee laxity. ACL injury is frequent in active young people, especially athletes involved in pivoting and contact sports. ACL reconstruction is the gold standard for the treatment of such patients who wish to return to sport and unrestricted physical activities. It is estimated that in the United States more than 300,000 ACL reconstructions are performed each year, a figure that gives a clear idea of the importance of this procedure in the field of orthopaedic and sports traumatology.

At the current state of the art in ACL reconstruction, multiple techniques have been presented but none has shown clearly defined and improved results. One of the main issues concerns the choice of graft. Bonepatellar tendon-bone (BPTB), hamstrings and quadriceps tendon are the most widely used autograft tissues. However, the possibility of donor site morbidity, especially when the extensor apparatus is involved, is 
a serious concern, as this can compromise the results of a well-performed reconstruction $(1,2)$.

The absence of graft harvesting when using allograft tissues, on the other hand, is an appealing feature that, by minimizing the risk of complications related to donor site morbidity, can improve the results of ACL reconstruction. However, this approach, too, raises serious concerns that have limited the worldwide adoption of ACL reconstruction with allografts, namely the risk of transmission of hepatitis, HIV and other diseases, ethical and religious issues, the ubiquitous tissue issues of consistent quality and availability, and the problem of the slower "ligamentization" process (3, 4). Consequently, the use of allograft tissues has been confined mostly to revision and multi-ligament procedures. In the past, in order to overcome these problems, various attempts have been made to develop ACL reconstruction devices with a combination of materials, which presented biological challenges. Synthetic devices, such as Dacron, Kevlar and carbon fiber showed some initial clinical success but ultimately failed because of inappropriate initial biomechanical properties, material fatigue profiles, and production of debris due to wear (5-8). The abraded synthetic particles collected in the joint space, lymph nodes and other tissues, leading to a chronic inflammatory response (9).

\section{Xenograft and immunological response}

The concept of using xenograft tissue, defined as graft tissue from one species and destined for implantation in an unlike species, was introduced in order to try to overcome the mechanical and biological concerns associated with synthetic materials and the safety and quality concerns and availability problems of allograft tissue. In the 1980s, a bovine BPTB bioprosthetic based on the glutaraldehyde crosslink chemistry used to produce porcine heart valves was proposed and used clinically, but the results were unsuccessful. This was mainly due to poor biocompatibility attributed to excess glutaraldehyde, insufficient biomechanical properties of the graft prior to implantation, and a lack of host integration (10).

The major challenge for transplantation of xenograft animal tissue into humans has been immunological rejection, an acute rejection process due mainly to the presence of a carbohydrate antigen called $\alpha$-galactosyl $(\alpha-G a l)$ found in the cellular and extracellular structures of almost all mammals except for humans and Old World primates (11). Humans and Old World primates naturally produce anti- $\alpha-G$ al antibodies, which can result in acute rejection of transplanted xenograft tissue derived from other mammals (12).

Attempts have been made to clone knockout pigs, which lack the $\alpha$-Gal epitopes, and thus to eliminate the anti-Gal reaction towards grafts or organs such as heart and kidneys when these are implanted into monkeys (13-16). Another immunological problem concerns deposits of antibodies, defined as "anti-non-Gal antibodies", which have been shown to remain immunogenic in humans and monkeys transplanted with xenograft with no $\alpha$-Gal epitopes $(17,18)$.

\section{The Z-Lig® ACL device}

In an attempt to overcome or attenuate the immune response against porcine xenografts, the Z-Lig ${ }^{\circledR}$ device for ACL reconstruction procedures was developed applying the Z-Process ${ }^{\circledR}$ (Aperion Biologics Inc, San Antonio, Texas, USA). The Z-Process ${ }^{\circledR}$ is used to control the immune response normally associated with $\alpha$ Gal and non-Gal epitopes.

This process is exploited to produce graft configurations, tendons with or without bone blocks, from animal tissue, which are consistent with what has normally been sourced from human tissue. In the current study, the porcine bone-patellar tendon graft was harvested and processed to overcome anti-Gal-mediated rejection and attenuate other immunological recognition in humans, while ensuring sterility, viral inactivation and mechanical properties appropriate for an ACL reconstruction device.

The four basic processing steps necessary to produce the device are:

1) tissue preparation and cleaning;

2) enzymatic elimination of $\alpha$-Gal epitopes from the porcine ligament using a solution containing recombinant $\alpha$-galactosidase;

3) stabilization with low-level crosslinking using a solution of $0.1 \%$ glutaraldehyde, in order to attenuate the immune response and slow anti-non-Gal infiltration, thereby preventing it from destroying 
the pig xenograft, and allowing for an appropriate "ligamentization"; 4) irradiation by E-beam (17.8 kGy) for final sterilization, and storage at temperatures below $-50^{\circ} \mathrm{C}$.

This study utilized a bone-tendon construct with a total product length of approximately $10 \mathrm{~cm}$ and a width of $1 \mathrm{~cm}$ sourced from a porcine patellar tendon graft, provided with two bone plugs, one sized for fixation and the other sized to assist in graft passage (Fig. 1), packed in double sterile pouches (Fig. 2). The Z-Lig ${ }^{\circledR}$ device has been tested in skeletally mature monkeys and gave interesting and promising results (19). The study looked at three areas to assess safety and performance: serological analysis to identify the immune response, mechanical testing to assess performance, and histopathological analysis to assess biological response and remodeling. At the twelve-month gross observation, the porcine xenograft was incorporated by the host as a functional graft, with re-establishment of the synovial sheath and proximal attachment vascularization. Moreover, there were no signs of degenerative articular changes or adverse synovial reaction. Similar findings were reported on histological analysis, which also demonstrated graft and bone-tohost cancellous bone consolidation inside the bone tunnels. The porcine graft underwent advanced maturation and remodeling, experiencing a "ligamentization" process similar to that undergone by rhesus allografts implanted in the control group. From the biomechanical point of view, the ultimate load to failure increased over time, at a rate not substantially different from that shown by the rhesus allograft. Finally, no adverse findings emerged from standard clinical chemistry, serolog- ical, and hematological panels. The anti-Gal titer was $95 \%$ lower than in a control group treated with porcine graft that was not treated with the $\alpha$-galactosidase process. However, the Authors detected a clinically irrelevant presence of anti-Gal and anti-non-Gal titer in the post-implantation period, but a progressive decrease was noted after nine and twelve months.

The positive results and the safety profile reported in the animal model were replicated in the first clinical trial involving humans (20). Ten patients with acute or chronic ACL injury were treated with ACL reconstruction using the Z-Lig ${ }^{\circledR}$ device. Four patients did not reach the final follow-up due to non-device-related complications during the study. At the 24 -month postoperative evaluation, five of the six evaluable subjects presented with functional grafts that were satisfactory on a variety of standard orthopaedic tests, and also showed no evidence of knee effusion. One of the six subjects presented with tibial bone plug loosening and had his xenograft removed. Blood chemistry and serology results of all patients were within acceptable normal ranges at the 24-month time point. These patients also showed a very low level of anti-Gal antibodies, as demonstrated by ELISA testing. The quantity of antinon-Gal antibodies, in line with what was observed in the animal studies, showed increased levels post transplantation. However, the anti-non-Gal activity decreased after 12 months and returned to pre-transplantation levels. Finally, histological sections of the xenografts showed the "ligamentization" process, consisting of infiltration of inflammatory cells and fibroblasts, and subsequently the production of newly synthesized collagen. One of the original Z-lig patients

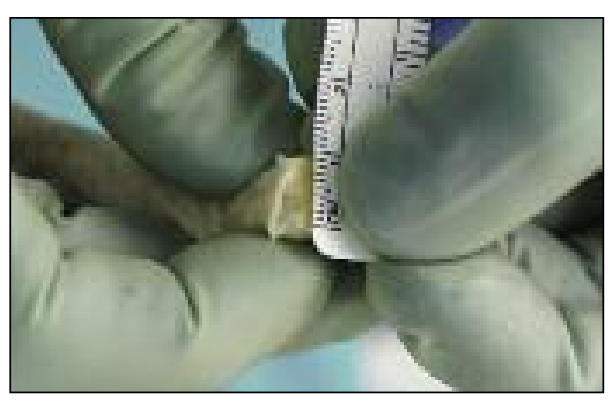

Fig. 1. The porcine patellar tendon used in this study was provided with one standard sized bone plug (length $25 \mathrm{~mm}$, diameter $10 \mathrm{~mm}$ ) on one end of the graft for solid bone-to-bone fixation inside the femoral tunnel and a smaller accessory bone plug (length $10 \mathrm{~mm}$, diameter $5 \mathrm{~mm}$ ) to facilitate graft passage.

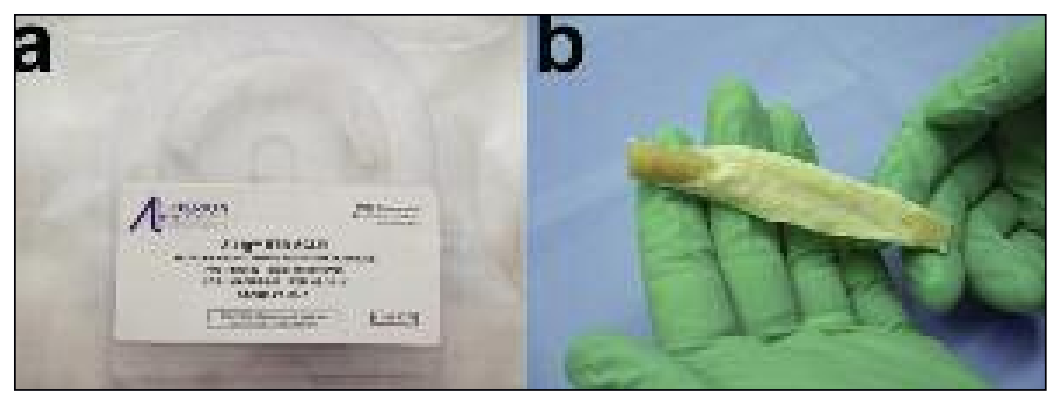

Fig. 2. The Z-Lig $₫$ device is provided already shaped, sized and ready for implantation, sealed in a sterile package. 
was still able to participate in ski racing and continued to race successfully until five years post implantation, and today is a professional ski instructor. These previous findings demonstrate that ACL reconstruction with porcine xenografts is a safe procedure.

Due to these compelling studies, an international multicenter prospective, randomized, blinded and controlled study, involving Italy, Spain, South Africa, Denmark and Belgium, was conducted on a wider patient population in order to confirm the safety profile and to compare the performance with respect to reconstruction using an allograft. The 24-month follow-up was completed and additional overall study results are forthcoming. Meanwhile, the Z-Lig ${ }^{\circledR}$ recently received $\mathrm{CE}$ mark approval for commercial use in selected countries.

\section{Surgical procedure}

The processed porcine BPTB graft can be implanted in patients with a ruptured ACL through a procedure identical to ACL reconstruction with autograft.

Standard arthroscopy is performed, and cartilage or meniscal injuries are addressed as needed. After removal from the freezer, the Z-Lig ${ }^{\circledR}$ sterile package is opened, and the device prepared. The xenograft is already pre-prepared, i.e. shaped and sized to meet standard clinical practice, so it requires only minimal preparation compared to allograft tissue. A drill is used to prepare a small hole in both bone plugs, in order to allow the placement of two passing sutures. Moreover, the part of the xenograft that will be placed in the tibial tunnel is whipstitched for $2-3 \mathrm{~cm}$ in order to ease final graft pulling and minimize the potential

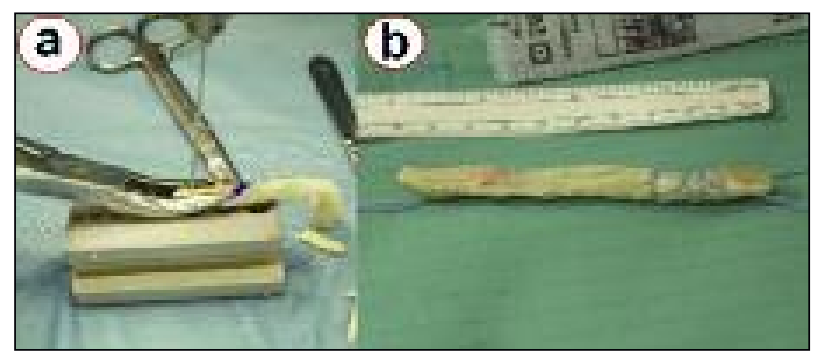

Fig. 3. The xenograft requires only minimal preparation: two holes are drilled in the bone plugs in order to allow the placement of two passing sutures (a), while the part of the tendon that should be placed in the tibial tunnel is whipstitched to improve screw purchase and minimize graft damage during screw insertion (b). for graft damage when it is used with interference screw fixation (Fig. 3). Once prepared, the graft is soaked in a solution containing rifampicin antibiotic. A 9-10-mm tibial tunnel is prepared by overreaming a guide pin directed from the medial aspect of the tibia to the posterior part of the anatomical ACL insertion site. Subsequently, a Beath pin is placed in the anatomical insertion of the posterolateral bundle of the ACL (Fig. 4). After reaming a half tunnel (25-30 $\mathrm{mm}$ long with a diameter of $9-10 \mathrm{~mm}$ ), the Beath pin is loaded with a passing suture and extracted from the lateral aspect of the thigh, pulling the suture through the femoral tunnel. A clamp is introduced through the tibial tunnel, and the previously placed suture is grasped and retrieved through the tunnel to the medial aspect of the tibia. At this point, the looped suture exiting from the tibial tunnel is loaded with the suture placed in the xenograft femoral bone plug. Pulling the suture that exits from the femoral tunnel, the graft is passed into the tibial tunnel, intra-articularly, and then into the femoral tunnel (Fig. 5). Applying tension to the suture, a metal interference screw is placed in the femoral tunnel to secure the graft. The graft is now tensioned with $5-10 \mathrm{~kg}$ of force with the suture attached to the tibial side of the graft. While tensioning the graft, the knee is moved through a full range of motion for 10-20 cycles. The goal is to reduce or eliminate any laxity present in the graft. While maintaining adequate tension on the graft with the knee bent at $30^{\circ}$ and with the tibia pushed backward mimicking posterior drawer, the tibial side of the graft is secured in the tunnel with another metal screw.

At the end of the procedure, drain suction is placed and the skin is closed. Rehabilitation and return to sporting activity did not substantially differ from what is seen with standard ACL reconstruction protocols.

\section{The Italian experience}

The II Orthopaedic and Traumatology Clinic of the Rizzoli Orthopaedic Institute was chosen along with other sites located worldwide to test the Z-Lig ${ }^{\circledR}$ device within an international multicenter study. Our clinic is the first center to test the device in Italy and the only Italian center participating in the study. In accordance with a strictly controlled and blinded study design, the data regarding the type of graft 
implanted remained blinded until the 24-month follow-up. At the end of the blinded period, three patients of the limited Italian study population were found to have received a xenograft.

The three patients were two males and one female, with an age ranging between 21 and 51 years. The ACL lesion was chronic in two cases and involved two right knees and one left knee. One patient was an amateur basketball player and another an amateur indoor soccer player.

Surgery was performed arthroscopically in all cases. The preparation of the graft required between 10 and 15 minutes to complete and was performed as described previously; minor trimming of the device was performed to obtain the final sizing and address surgeon preferences. The graft preparation was faster and easier than experienced with conventional allograft tendons.

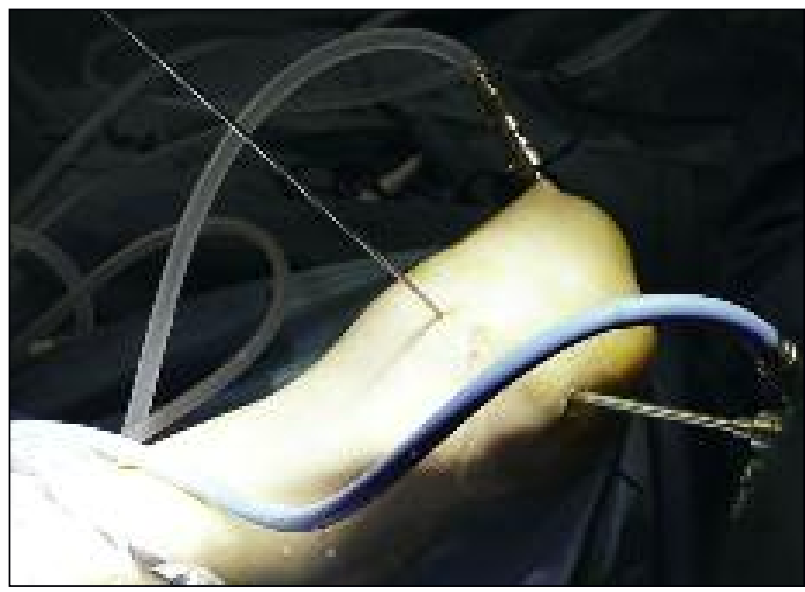

Fig. 4. A Beath pin is placed in the anatomical insertion of the posterolateral bundle of the ACL and directed through the lateral aspect of the thigh. The pin will be loaded with a passing suture.

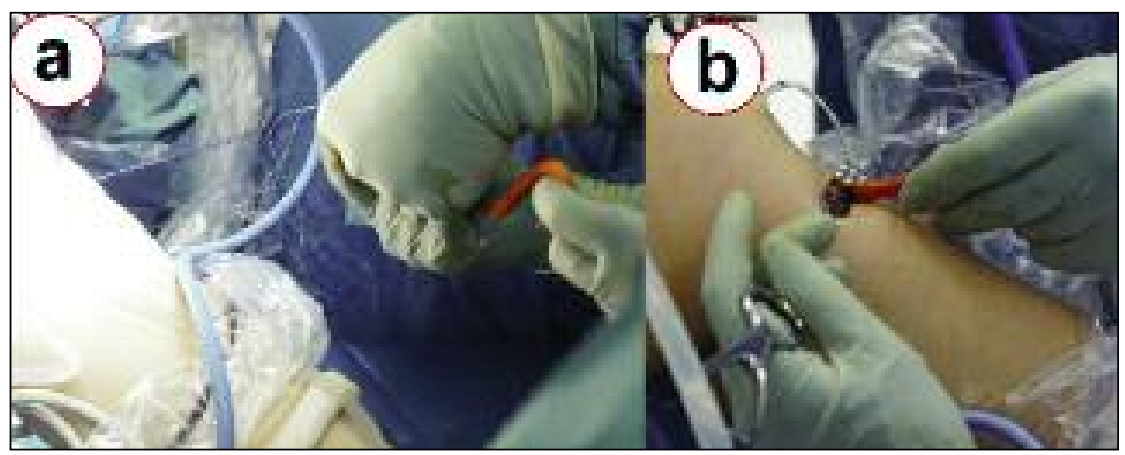

Fig. 5. The looped suture that exits from the tibial tunnel is loaded with the suture placed in the xenograft femoral bone plug (a). Therefore, pulling the suture that exits from the femoral tunnel allows the graft to be passed into the tibial tunnel (b), the knee joint, and finally the femoral tunnel.
During the graft preparation the tendon and bone quality was found to be much better than that seen with allografts. The whole surgical procedure took less than 60 minutes. Two patients underwent concomitant meniscectomy (1 medial and 1 lateral) while one patient underwent a lateral meniscus suture repair for a posterior horn tear.

Tunnel positions were consistent with an anatomical technique and the size of femoral and tibial tunnels was $9 \mathrm{~mm}$ in all cases; graft fixation was achieved with metal and resorbable interference screws on the femoral and tibial side respectively.

Clinically, all the patients showed increased stability from the early six-month evaluation through to the final 24-month follow-up; KT-1000, Objective IKDC and pivot-shift maneuver were used for the objective evaluations. All the patients presented normal or near normal values.

Functional testing using the one-legged hop test also indicated a functional return to a high level versus the unoperated knee at 12 and 24 months after surgery. From the subjective point of view, an increase in Tegner activity level was noted at six months after surgery and the Subjective IKDC score also reached near normal values in the course of the follow-up. Satisfaction was rated at between 9 and 10 on a 10point scale, and all the patients stated that they would be willing to undergo the procedure again.

Radiographs showed good tunnel and screw positioning, and no gross progression of osteoarthritis at the 24-month follow-up. No gross tunnel enlargement or changes of bone opacity were detected.

MRI evaluation showed a hypointense graft on T2weighted images. The graft was well represented showing intact fibers, no lesions and a normal cross-sectional area (Fig. 6). No signs of intra-articular reaction or pathology were detected; furthermore, a reduction of synovial thickness, bone edema and internal effusion were noted, which highlighted the absence of an inflammatory reaction. Also the MRI confirmed the good status of the tibial and femoral tunnels and the bone-bone interface.

Blood samples obtained at the 24month follow-up did not indicate a 
major presence of anti-Gal and antinon-Gal antibodies.

This finding was consistent with an initial elevation followed by a return to baseline over the 24-month period, and it confirmed the safety profile of the device as suggested in the preclinical animal models and pilot human clinical safety study.

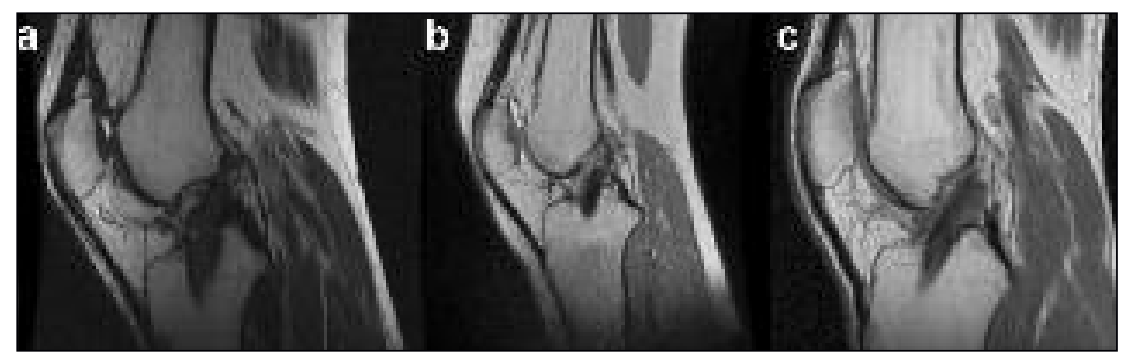

Fig. 6. Two-year follow-up MRI of the three patients that received the Z-Lig® device. MRI signal show good status of the graft, with no signs of inflammatory process.

\section{Conclusions}

The positive and encouraging results of the animal and human studies performed to evaluate the safety and efficacy of ACL reconstruction with a porcine xenograft could make the Z-Lig ${ }^{\circledR}$ a definitive option, extending the range of graft options open to clinicians. The treatment with $\alpha$-galactosidase and subsequent stabilization of the animal tissue graft serve to control and almost eliminate the potential for an immunological reaction, thus minimizing the risk of rejection and complications that might adversely affect patients' health. The device maintains function and provides stability during the time required for remodeling and "ligamentization". This study presents the limited experience of the Italian center with this novel implant. The results of the multicenter, international controlled study will provide a fuller understanding of the potential efficacy and utility of the Z-Lig ${ }^{\circledR}$ device compared to the current standards of care.

\section{References}

1. Christen B, Jakob RP. Fractures associated with patellar ligament grafts in cruciate ligament surgery. J Bone Joint Surg Br. 1992;74: 617-619.

2. Kohn D, Sander-Beuermann A. Donor-site morbidity after harvest of a bone-tendon-bone patellar tendon autograft. Knee Surg Sports Traumatol Arthrosc. 1994; 2:219-223.

3. Butler DL. Kappa Delta Award paper. Anterior cruciate ligament: its normal response and replacement. J Orthop Res. 1989;7:910-921.

4. Amiel S, Kuiper S. Experimental studies on anterior cruciate ligament grafts. In: Knee Ligaments: Structure, Function, Injury and Repair. Daniel D, ed. Baltimore: Raven Press. 1990;379-388

5. Bolton CW, Bruchman WC. The GORE-TEX expanded polytetrafluoroethylene prosthetic ligament. An in vitro and in vivo evaluation Clin Orthop Relat Res. 1985;(196):202-213.

6. Lukianov AV, Richmond JC, Barrett GR, Gillquist J. A multicenter study on the results of anterior cruciate ligament reconstruction using a Dacron ligament prosthesis in "salvage" cases. Am J Sports Med. 1989;17: 380-386.
7. Schindhelm K, Rogers GJ, Milthorpe BK, et al. Autograft and Leeds-Keio reconstructions of the ovine anterior cruciate ligament. Clin Orthop Relat Res. 1991;(267): 278-293.

8. Weiss AB, Blazina ME, Goldstein AR, Alexander H. Ligament replacement with an absorbable copolymer carbon fiber scaffold-early clinical experience. Clin Orthop Relat Res. 1985,(196):77-85.

9. Margevicius KJ, Claes LE, Dürselen L, Hanselmann K. Identification and distribution of synthetic ligament wear particles in sheep. J Biomed Mater Res. 1996,31:319-328.

10. Buhler L, Xu Y, Li W, Zhu A, Cooper DK. An investigation of the specificity of induced anti-pig antibodies in baboons. Xenotransplantation. 2003;10:88-93.

11. Galili U. Interaction of the natural anti-Gal antibody with alpha-galactosyl epitopes: a major obstacle for xenotransplantation in humans. Immunol Today. 1993;14:480-482.

12. Galili U, LaTemple DC, Walgenbach AW, Stone KR. Porcine and bovine cartilage transplants in cynomolgus monkey: II. Changes in anti-Gal response during chronic rejection. Transplantation. 1997;63:646-651.

13. Lai L, Kolber-Simonds D, Park KW, et al. Production of alpha-1,3-galactosyl transferase knockout pigs by nuclear transfer cloning. Science. 2002;295:1089-1092.

14. Phelps CJ, Koike C, Vaught TD, et al. Production of alpha 1,3-galactosyltransferase-deficient pigs. Science. 2003;299: 411-414.

15. Kuwaki K, Tseng YL, Dor FJ, et al. Heart transplantation in baboons using alpha1,3-galactosyltransferase gene-knockout pigs as donors: initial experience. Nat Med. 2005;11:29-31.

16. Yamada K, Yazawa K, Shimizu A, et al. Marked prolongation of porcine renal xenograft survival in baboons through the use of alpha1,3-galactosyl transferase gene-knockout donors and the cotransplantation of vascularized thymic tissue. Nat Med. 2005;11:32-34.

17. Galili U, Chen ZC, Tanemura M, et al. Induced antibody response in xenograft recipients. GRAFT. 2001;4:32-35.

18. Stone KR, Ayala G, Goldstein J, et al. Porcine cartilage transplants in the cynomolgus monkey. III. Transplantation of alpha-galactosidase-treated porcine cartilage. Transplantation. 1998;65:1577-1583.

19. Stone KR, Walgenbach AW, Turek TJ, Somers DL, Wicomb W, Galili U. Anterior cruciate ligament reconstruction with a porcine xenograft: a serologic, histologic, and biomechanical study in primates. Arthroscopy. 2007;23:411-419.

20. Stone KR, Abdel-Motal UM, Walgenbach AW, et al. Replacement of human anterior cruciate ligaments with pig ligaments: a model for anti-non-gal antibody response in long-term xenotransplantation. Transplantation. 2007;83: 211-219. 